\title{
Dynamic Reserve and Transmission Capacity Allocation in Wind-Dominated Power Systems
}

Viafora, Nicola; Delikaraoglou, Stefanos; Pinson, Pierre; Hug, Gabriela; Holbøll, Joachim

Published in:

IEEE Transactions on Power Systems

Link to article, DOI:

10.1109/TPWRS.2020.3043225

Publication date:

2021

Document Version

Peer reviewed version

Link back to DTU Orbit

Citation (APA):

Viafora, N., Delikaraoglou, S., Pinson, P., Hug, G., \& Holbøll, J. (2021). Dynamic Reserve and Transmission Capacity Allocation in Wind-Dominated Power Systems. IEEE Transactions on Power Systems, 36(4), 30173028. https://doi.org/10.1109/TPWRS.2020.3043225

\section{General rights}

Copyright and moral rights for the publications made accessible in the public portal are retained by the authors and/or other copyright owners and it is a condition of accessing publications that users recognise and abide by the legal requirements associated with these rights.

- Users may download and print one copy of any publication from the public portal for the purpose of private study or research.

- You may not further distribute the material or use it for any profit-making activity or commercial gain

- You may freely distribute the URL identifying the publication in the public portal 


\title{
Dynamic Reserve and Transmission Capacity Allocation in Wind-Dominated Power Systems
}

\author{
Nicola Viafora, Student Member, IEEE, Stefanos Delikaraoglou, Member, IEEE, Pierre Pinson, Fellow, IEEE, \\ Gabriela Hug, Senior Member, IEEE, Joachim Holbøll, Senior Member, IEEE
}

\begin{abstract}
The large shares of wind power generation in electricity markets motivate higher levels of operating reserves. However, current reserve sizing practices fail to account for important topological aspects that might hinder their deployment, thus resulting in high operating costs. Zonal reserve procurement mitigates such inefficiencies, however, the way the zones are defined is still open to interpretation. This paper challenges the efficiency of predetermined zonal setups that neglect the location of stochastic power production in the system, as well as the availability, cost and accessibility of flexible generating units. To this end, we propose a novel reserve procurement approach, formulated as a two-stage stochastic bilevel model, in which the upper level identifies a number of contiguous reserve zones using dynamic grid partitioning and sets zonal requirements based on the total expected operating costs. Using two standard IEEE reliability test cases, we show how the efficient partitioning of reserve zones can reduce expected system cost and promote the integration of stochastic renewables.
\end{abstract}

Index Terms-Zonal reserve requirements, bilevel optimization, stochastic programming, grid partitioning, transmission capacity allocation.

A. Sets and Indices

\section{NOMENCLATURE}
$n \in \mathcal{N}$
Set of nodes.
$\ell \in \mathcal{L}$
Set of transmission lines.
$g \in \mathcal{G}$
Set of conventional generators.
$j \in \mathcal{J}$
Set of wind power generators.
$z \in \mathcal{Z}$
Set of partitions.
$s \in \mathcal{S}$
Set of scenarios.

\section{B. Parameters}

$\bar{P}_{g}, \underline{P}_{g}$

$F_{\ell}$

Max/min generator's output.

$R_{g}^{+}, R_{g}^{-} \quad \mathrm{Up} /$ down reserve capacity offer.

$\Lambda^{+}, \Lambda^{-} \quad$ Up/down deterministic reserve requirement.

$C_{g}, C_{g}^{+/-}$Generation and up/down reserve cost.

$C^{\text {sh }}, C^{\text {ct }} \quad$ Load shedding and wind curtailment cost.

$\boldsymbol{H} \quad$ Incidence matrices.

$M \quad$ Power transfer distribution factor matrix.

$D_{n}, \boldsymbol{D} \quad$ Nodal load demand.

$\pi_{s} \quad$ Probability of scenario.

$\widehat{W}_{j}, W_{j, s}$ Wind power point forecast and realization.

$\chi$

Maximum withdrawn capacity from day-ahaed market

\section{Decision variables}

$\begin{array}{ll}x_{n, z} & \text { Binary variable for grid partitioning. } \\ y_{z} & \text { Number of nodes per zone. } \\ \varphi_{\ell, z} & \text { Flowing units on line } \ell \text { in zone } z \text { for expressing } \\ & \text { zone connectivity. }\end{array}$

$c_{n, z}$

$r_{g, z}^{+}, r_{g, z}^{-}$

$\lambda_{z}^{+}, \lambda_{z}^{-}$

$\hat{f}_{\ell}, f_{\ell, s}$

$p_{g}, \boldsymbol{p}$

$p_{g, s}^{+}, p_{g, s}^{-}$

$w_{j}, w_{j, s}^{\mathrm{ct}}$

$d_{n, s}^{\text {sh }}$

$w_{j, s}^{\mathrm{ct}}$

$\Gamma_{\ell}$
Root node selection.

Up/down procured reserve.

Up/down zonal reserve requirement.

Expected power flow and realization in scen. $s$.

Day-ahead dispatch of conventional generators.

Up/down reserve deployment per scenario.

Scheduled and curtailed wind power.

Nodal load shedding per scenario.

Wind power curtailment per scenario.

Capacity allocation margin at day-ahead market.

\section{INTRODUCTION}

Several studies indicate that high shares of wind power generation require significantly more operating reserves to accommodate the uncertainty and the variability arising from forecast errors and inherent fluctuations in the wind regime [1]. However, simply increasing the reserve capacity requirements does not guarantee that the system will have access to sufficient flexible resources during real-time operation, since the existing reserve capacity market is myopic about the grid topology limitations. As a result, in cases when operating reserves cannot be delivered due to network congestions, system operators have to resort to more expensive corrective actions, such as wind curtailment and load shedding.

An implicit way to account for network limitations is to consider a zonal representation of the system. This approximation allows system operators to differentiate zonal reserve requirements based on expected congestion patterns and the location of stochastic power production. The impact of network limitations on the procurement of zonal reserve capacity has been investigated in [2] and [3], whereas authors in [4] and [5] rely on injection shift factors to evaluate potential congestions that would limit the deliverability of reserves. Despite being an approximation of the true network topology, the zonal splitting approach is readily compatible with the current market structure and allows to convey to the reserve market more complete information about the balancing needs of the system at specific locations. This is a fundamental property of the more advanced energy and reserves co-optimization models based on two-stage stochastic programming. This methodology is adopted by authors of [6] for considering contingencies and their associated probabilities on the reserve procurement process, whereas [7] and [8] focus on wind power uncertainty. While these approaches minimize by definition the total expected costs of the system, this result comes at the expense of violating the cost recovery and revenue adequacy properties for some uncertainty realizations [9]. 
This latter consideration has motivated several studies to use a stochastic bilevel programming approach that preserves the existing market structure and its desirable economic properties not only in expectation, but for every uncertainty outcome. Authors in [9] adopt this framework for optimally dispatching wind power in an energy-only market, whereas authors in [10] employ an analogous approach to define the optimal reserve requirements in view of wind power uncertainty. In a similar vein, [11] extends this model to account for the allocation of cross-border transmission capacity between energy and reserves. Although these models have shown to improve the total expected cost in a sequential market-clearing architecture, they still lack the ability to optimally position reserves in the system, as the ideal stochastic model does. This stems from the merit-order principle enforced by the existing market design, which restricts the procurement of reserves from the cheapest generators, regardless of their location in the system.

The aforementioned studies considered either a single zone or a predefined zonal setup for the reserve procurement. This paper proposes a novel Zonal Preemptive model, which does not only define the zonal reserve requirements, but it also considers the zone boundaries as decision variables. The main goal of this model is to improve the positioning of reserves in the system, while remaining compatible with the current market structure. In this work, we build upon [10] and [11] and we embed grid partitioning algorithms in the stochastic bilevel problem in order to identify a number of zonal reserve markets to be cleared independently. Grid partitioning algorithms have been used already in power system research for intentional islanding studies in [12] and [13]. However, to the best of our knowledge, this is the first attempt to rely on them for setting zonal reserve requirements. The proposed approach can be used as a decision-support tool by grid operators taking into consideration the location and forecast uncertainty of stochastic power production, the asymmetry of balancing costs as well as the reserve deliverability issues that may arise in real-time operation due to network constraints.

While zonal reserve allocation is not a novel concept, the way the zones are defined is still open to interpretation. Existing studies base the partitioning of the system on heuristic methods that consider: active and reactive power flow sensitivities [14]; data-driven clustering techniques [15]; weighted power transfer distribution factors (PTDFs) [16]; reserve market clearing prices [17] or simply use pre-defined partitions [18] that can be based on geographical boundaries or ownership. Alternatively, authors in [19] and [20] propose a method to disqualify zonal reserves if transmission constraints are likely to limit their deliverability and evaluate their performance on the day-ahead market. On the contrary, the proposed approach relies on a novel partitioning scheme that is solely driven by the total expected system costs and a high quality description of system uncertainties in the form of scenarios, instead of a proxy metric that can only partially describe the system states and the market outcomes under different operating conditions.

In addition to the reserve zones configuration our formulation is able to perform an optimal allocation of the inter-zonal transmission capacity between energy and reserve services as in [11]. Setting aside part of the transmission capacity for reserve exchange between adjacent zones has shown to lower the total operating costs [21] and it is a measure considered in practice by grid operators [22]. In view of the dynamic configuration of reserve zones, our methodology is capable to redefine accordingly the set of cross-zonal lines eligible for reserve exchange, by adapting the grid partitioning constraints. Our simulation results are showcased based on both IEEE RTS-24 and IEEE RTS-96 systems, where we benchmark our methodology against a sequential approach, the stochastic energy and reserve co-optimization and the stochastic bilevel with a single or predefined zones.

The main contributions of the paper are the following:

(i) We combine a grid partitioning algorithm with a stochastic bilevel optimization problem, introducing an additional degree of freedom in the allocation of zonal reserve capacity, i.e., the definition of reserve zones boundaries.

(ii) The proposed methodology allows to further approximate the ideal stochastic energy and reserve co-optimization model, without resorting to generator-specific reserve procurement that violates the merit-order principle. Instead, by dynamically defining reserve zones, reserve requirements and cross-zonal transmission capacity, our methodology is able to improve the deliverability of reserves, while remaining compatible with existing European market structure.

(iii) Finally, our work contributes to the ongoing discussion on the integration of European reserve and balancing markets in [23], [24] and [25] pointing to the need for a dynamic redefinition of reserve zones as soon as the procurement of flexible generation capacity extends beyond national borders and embraces a regional or continental scale.

The remainder of the paper is organized as follows. The existing reserve procurement and dispatch models of the current sequential market architecture as well as the stochastic energy and reserves co-optimization model that establishes an ideal benchmark in terms of expected system cost are reviewed in Section II. The proposed Zonal Preemptive model is presented in Section III, whereas the reformulation of the bilevel model as a tractable Mixed-Integer Linear Program (MILP) and a more computationally efficient solution scheme based on Benders decomposition are presented in Section IV. Lastly, Section V elaborates on simulation results based on the IEEE RTS-24 and IEEE RTS-96 test systems and Section VI concludes the paper.

\section{Reserve Procurement And Dispatch Models}

We first provide the mathematical formulation of the existing European market design, based on the sequential clearing of the reserve capacity, day-ahead energy and balancing markets. We then provide a compact formulation of the stochastic energy and reserve co-optimization model, emphasizing its main differences compared to the sequential approach.

\section{A. Sequential Approach}

Let $\Lambda^{+}$and $\Lambda^{-}$indicate the upward and downward reserve requirements. These are provided as exogenous parameters to the reserve market clearing algorithm that is formulated as 


$$
\begin{array}{ll}
\min _{\Xi_{\mathrm{R}}} & \mathcal{C}_{\mathrm{R}}=\sum_{g \in \mathcal{G}}\left(C_{g}^{+} r_{g}^{+}+C_{g}^{-} r_{g}^{-}\right) \\
\text {s.t. } & \sum_{g \in \mathcal{G}} r_{g}^{+} \geq \Lambda^{+}, \quad \sum_{g \in \mathcal{G}} r_{g}^{-} \geq \Lambda^{-}, \\
& 0 \leq r_{g}^{+} \leq R_{g}^{+}, \quad 0 \leq r_{g}^{-} \leq R_{g}^{-}, \quad \forall g \in \mathcal{G},
\end{array}
$$

where $\Xi_{\mathrm{R}}=\left\{r_{g}^{+}, r_{g}^{-}, \forall g\right\}$ is the set of optimization variables, i.e., up- and downward reserve capacity procured from each generator. Constraints $(1 \mathrm{~b})$ guarantee that the pre-determined reserve requirements $\Lambda$ are met, whereas (1c) limit the amount of reserve that can be procured to generators' capacity offers.

Having reserve capacity procurement $r_{g}^{+, *}$ and $r_{g}^{-, *}$ from model (1) as fixed parameters, the optimal day-ahead energy schedule for conventional $p_{g}$ and stochastic $w_{j}$ generators is obtained solving the following problem

$$
\begin{array}{lll}
\min _{\Xi_{\mathrm{D}}} & \mathcal{C}_{\mathrm{D}}=\sum_{g \in \mathcal{G}} C_{g} p_{g} & \\
\text { s.t. } & \sum_{g \in \mathcal{G}} p_{g}+\sum_{j \in \mathcal{J}} w_{j}=\sum_{n \in \mathcal{N}} D_{n}, & \\
& \underline{P}_{g}+r_{g}^{-, *} \leq p_{g} \leq \bar{P}_{g}-r_{g}^{+, *}, & \\
& 0 \leq w_{j} \leq \widehat{W}_{j}, & \forall j \in \mathcal{G}, \\
& \widehat{f}_{\ell}=\boldsymbol{M}_{(\ell, \cdot)}\left(\boldsymbol{H}_{\mathrm{G}}^{\top} \boldsymbol{p}+\boldsymbol{H}_{\mathrm{J}}^{\top} \boldsymbol{w}-\boldsymbol{D}\right), & \forall \ell \in \mathcal{L}, \\
& -F_{\ell} \leq \widehat{f}_{\ell} \leq F_{\ell}, & \forall \ell \in \mathcal{L}
\end{array}
$$

where $\Xi_{\mathrm{D}}=\left\{p_{g}, \forall g ; w_{j}, \forall j\right\}$ is the set of optimization variables, comprising day-ahead energy quantities for each conventional and stochastic generator. The day-ahead power balance is enforced by constraint (2b), whereas the production of conventional units is bounded by the minimum and maximum generation limits and procured reserves in constraint (2c). Without loss of generality, stochastic producers are assumed to be wind power generators only, whose dispatch is limited to the available point forecast $\widehat{W}_{j}$ in constraint (2d). While this paper focuses on wind power generation only, the formulations included in this section and the proposed methodology can include additional sources of uncertainty, such as solar power or uncertain demand. Employing a DC network approximation, power flows are modelled by (2e) using the PTDF matrix $\boldsymbol{M}$ and are in turn restricted by the corresponding transmission capacity limits in (2f). Appropriate incidence matrices $\boldsymbol{H}_{\mathrm{G}}$ and $\boldsymbol{H}_{\mathrm{J}}$ map conventional and stochastic generators to the respective buses in the system.

Approaching the hour of the delivery when wind power realization $W_{j, s^{\prime}}$ is known, the balancing market is cleared using the following model to ensure that any deviation from the day-ahead schedule $p_{g}^{*}, w_{j}^{*}$ is balanced by appropriate redispatch actions for the uncertainty realization $s=s^{\prime}$.

$$
\begin{aligned}
\min _{\Xi_{\mathrm{B}, s^{\prime}}} \mathcal{C}_{\mathrm{B}, s^{\prime}} & =\sum_{g \in \mathcal{G}} C_{g}\left(p_{g, s^{\prime}}^{+}-p_{g, s^{\prime}}^{-}\right) \\
& +\sum_{j \in \mathcal{J}} C^{\mathrm{ct}} w_{j, s^{\prime}}^{\mathrm{ct}}+\sum_{n \in \mathcal{N}} C^{\mathrm{sh}} d_{n, s^{\prime}}^{\mathrm{sh}}
\end{aligned}
$$

s.t.

$\sum_{g \in \mathcal{G}}\left(p_{g, s^{\prime}}^{+}-p_{g, s^{\prime}}^{-}\right)+\sum_{j \in \mathcal{J}}\left(\Delta W_{j, s^{\prime}}-w_{j, s^{\prime}}^{\mathrm{ct}}\right)+\sum_{n \in \mathcal{N}} d_{n, s^{\prime}}^{\mathrm{sh}}=0$

$$
\begin{array}{ll}
0 \leq p_{g, s^{\prime}}^{+} \leq r_{g}^{+, *}, & \forall g \in \mathcal{G}, \quad(3 \mathrm{c}) \\
0 \leq p_{g, s^{\prime}}^{-} \leq r_{g}^{-, *}, & \forall g \in \mathcal{G},(3 \mathrm{~d}) \\
0 \leq d_{n, s^{\prime}}^{\text {sh }} \leq D_{n}, & \forall n \in \mathcal{N},(3 \mathrm{e}) \\
0 \leq w_{j, s^{\prime}}^{\mathrm{ct}} \leq W_{j, s^{\prime}}, & \forall j \in \mathcal{J},(3 \mathrm{f}) \\
-F_{\ell} \leq f_{\ell, s^{\prime}} \leq F_{\ell}, & \forall \ell \in \mathcal{L},(3 \mathrm{~g}) \\
f_{\ell, s^{\prime}}=\boldsymbol{M}_{(\ell, \cdot)}\left[\boldsymbol{H}_{\mathrm{G}}^{\top}\left(\boldsymbol{p}^{*}+\boldsymbol{p}_{s^{\prime}}^{+}-\boldsymbol{p}_{s^{\prime}}^{-}\right)\right. & \\
\left.+\boldsymbol{H}_{\mathrm{J}}^{\top}\left(\boldsymbol{W}_{s^{\prime}}-\boldsymbol{w}^{\mathrm{ct}}\right)-\left(\boldsymbol{D}-\boldsymbol{d}_{s^{\prime}}^{\mathrm{sh}}\right)\right], & \forall \ell \in \mathcal{L} \quad(3 \mathrm{~h})
\end{array}
$$

where $\Xi_{\mathrm{B}, s^{\prime}}=\left\{p_{g, s^{\prime}}^{+}, p_{g, s^{\prime}}^{-}, \forall g ; w_{j, s^{\prime}}^{\mathrm{ct}}, \forall j ; d_{n, s^{\prime}}^{\mathrm{sh}}, \forall n\right\}$ is the set of re-dispatch decisions, which comprises the deployment of upward and downward reserves, load shedding, wind spillage and $\Delta W_{j, s^{\prime}}=W_{j, s^{\prime}}-w_{j}^{*}$ denotes system imbalance. The objective function (3a) includes a cost $C_{g}$ for the activation of reserves from those generators that were cleared to provide reserves and have already received a capacity payment. Additionally, we assume that the grid operator has to face a cost $C^{\text {ct }}$ and $C^{\text {sh }}$ for wind power curtailment and load not supplied, respectively. Constraint (3b) is the real-time power balance, whereas constraints (3c)-(3d) limit the reserves activation to the procured values in (1). The use of corrective actions is limited by constraints (3e) and (3f), which model load shedding and wind curtailment, respectively. Finally, constraints $(3 \mathrm{~g})$ enforce power flow limits, where real-time power flows in each scenario are modelled in (3h).

\section{B. Stochastic Energy and Reserve Co-Optimization}

An improved method based on two-stage stochastic programming allows the grid operator to jointly co-optimize energy and reserves. In this framework, the first stage models reserve as well as day-ahead energy scheduling, whereas the second stage corresponds to the balancing market under each uncertainty realization contained in the scenario set $\mathcal{S}$. The stochastic energy and reserve co-optimization model is formulated as

$$
\begin{aligned}
& \min _{\Xi_{\mathrm{S}}} \mathcal{C}_{\mathrm{S}}=\mathcal{C}_{\mathrm{R}}+\mathcal{C}_{\mathrm{D}}+\sum_{s \in \mathcal{S}} \pi_{s} \mathcal{C}_{\mathrm{B}, s} \\
& \text { s.t. (1c), Reserve market } \\
& \text { (2b) - (2e), Day-ahead market } \\
& \text { (3b) - (3h), Balancing market, } \forall s \in \mathcal{S}
\end{aligned}
$$

where $\Xi_{S}=\left\{\Xi_{R} \cup \Xi_{D} \cup \Xi_{B, s}, \forall s\right\}$ is the set of optimization variables. The stochastic co-optimization of energy and reserves attains perfect temporal coordination, as opposed to the sequential model that separates the day-ahead and balancing decisions. Each generator is pre-positioned even out of merit order in a way that allows optimal delivery to the system in case of deviations from the day-ahead schedule. For this reason, we use the stochastic co-optimization approach as a benchmark to our proposed methodology, since it provides a lower bound to the total operating costs.

\section{Reserve and CAPacity Allocation Models}

This section introduces the concepts and the mathematical formulations that underpin the contributions of this work. The bilevel models in [10] and [11] are enhanced with a set of 
upper-level grid partitioning constraints described in III-A. These enable the operator to identify a pre-specified number of zones in the system, where zonal reserve markets can be cleared following the problem formulation in III-B.The model is complemented with a set of upper-level decision variables that account for the optimal allocation of transmission capacity between energy trading and re-dispatch actions in III-C. Finally, the full problem formulation of the proposed Zonal Preemptive methodology is presented in III-D.

\section{A. Grid Partitioning}

Let $\Theta=(\mathcal{N}, \mathcal{L})$ be a directed graph with $\mathcal{N}$ nodes and $\mathcal{L}$ edges describing the direct sequence equivalent topology of a power system. The partition of such a graph into $\mathcal{Z}$ connected sub-graphs or zones can be achieved by assigning as many binary variables $x_{n, z} \in\{0,1\}$ as the number of zones to each node. If node $n$ belongs to zone $z$, then $x_{n, z}=1$; otherwise $x_{n, z}=0$. Two important properties need to be satisfied in order to get the desired partition: (1) each node belongs to one and only zone; (2) the sub-graphs determined by the partition are connected, i.e., whichever two points are selected inside a zone, there always exists a path connecting them within the same zone. The first property is satisfied with

$$
\sum_{z \in \mathcal{Z}} x_{n, z}=1, \quad \forall n \in \mathcal{N}
$$

whereas, to achieve the second property, this paper adopts the single-commodity flow method presented in [12]. As discussed in [26], the relevant literature includes a variety of methods that ensure sub-graph connectivity in similar problems, however, the single-commodity flow has been selected here due to its simplicity and intuitive understanding. This method relies on flowing units, which bear no physical meaning, but allow to express the connectivity as the ability to reach all nodes in a zone, while staying within its boundaries. This method works by injecting $y_{z}$ units, i.e., as many as the number of nodes in the $z$-th zone, into a single arbitrary node of each sub-graph and enforcing

$$
y_{z}=\sum_{n \in \mathcal{N}} x_{n, z}, \quad \underline{y}_{z} \leq y_{z} \leq \bar{y}_{z}, \quad \forall z \in \mathcal{Z},
$$

where the quantity $y_{z}$ can be bounded by $\underline{y}_{z}$ and $\bar{y}_{z}$ in order to require a minimum or a maximum size of each zone in the system, respectively. A sub-graph is then connected if all the injected units can flow to the nodes in that sub-graph, without violating nodal flow balance and branch flow limit constraints. Nodal flow balance is expressed in a matrix notation as

$$
\boldsymbol{H}_{(\cdot, n)}^{\top} \boldsymbol{\varphi}_{(\cdot, z)}+c_{n, z} y_{z}=x_{n, z}, \quad \forall n \in \mathcal{N}, \quad \forall z \in \mathcal{Z},
$$

where $\boldsymbol{H}_{(\cdot, n)}$ indicates the $n$-th column of the branch incidence matrix, whose $\ell$-th value is 1 if line $\ell$ enters node $n,-1$ if it leaves it, or 0 otherwise. The flow of units injected in zone $z$ over all branches in the system is collected in $\varphi_{(\cdot, z)}$. Therefore, the scalar product $\boldsymbol{H}_{(\cdot, n)}^{\top} \boldsymbol{\varphi}_{(\cdot, z)}$ describes the net inor out-coming flow of units to or from node $n$ in zone $z$. The bilinear term $c_{n, z} y_{z}$ represents instead the injection of $y_{z}$ flow units into the root nodes defined by $c_{n, z}$, whereas the righthand-side acts as a sink, i.e., if node $n$ is included in zone $z$, it retains one unit.

Note that unlike [12], this modified version of the singlecommodity flow method does not require the root nodes $c_{n, z}$ to be pre-specified. This requirement would limit the degrees of freedom of the partitioning algorithm, as it relies on the choice of the initial nodes, from which the sub-graphs are generated. This last step is not necessary here, since $c_{n, z}$ is treated as a binary variable, which selects a node where the units are injected. The following constraints are added to ensure that the selected root nodes are mutually exclusive and that only one node per zone is selected as the root, i.e,

$$
\begin{array}{ll}
\sum_{z \in \mathcal{Z}} c_{n, z} \leq 1, & \forall n \in \mathcal{N}, \\
\sum_{n \in \mathcal{N}} c_{n, z}=1, & \forall z \in \mathcal{Z} .
\end{array}
$$

Finally, the branch flow limits are specifically defined to restrict the flow of units $\varphi_{\ell, z}$ to those lines that have both ends included in the same sub-graph. This aspect is modelled using the following constraints

$$
\begin{array}{lll}
-\Phi_{\mathrm{F}_{\ell, z}} \leq \varphi_{\ell, z} \leq \Phi_{\mathrm{F}_{\ell, z}}, & \forall \ell \in \mathcal{L}, & \forall z \in \mathcal{Z}, \\
-\Phi_{\mathrm{T}_{\ell, z}} \leq \varphi_{\ell, z} \leq \Phi_{\mathrm{T}_{\ell, z}}, & \forall \ell \in \mathcal{L}, & \forall z \in \mathcal{Z}, \\
\Phi_{\mathrm{F}_{\ell, z}}=y_{z}\left(\boldsymbol{H}_{\mathrm{F}_{(\ell, \cdot)}} \boldsymbol{x}_{(\cdot, z)}\right), & \forall \ell \in \mathcal{L}, \quad \forall z \in \mathcal{Z}, \\
\Phi_{\mathrm{T}_{\ell, z}}=y_{z}\left(\boldsymbol{H}_{\mathrm{T}_{(\ell, \cdot)}} \boldsymbol{x}_{(\cdot, z)}\right), & \forall \ell \in \mathcal{L}, \quad \forall z \in \mathcal{Z} .
\end{array}
$$

where $\boldsymbol{H}_{\mathrm{F}}$ and $\boldsymbol{H}_{\mathrm{T}}$ indicate "from" and "to" incidence matrices, respectively. For any given sub-graph, the maximum flow of units on each branch is bounded both by $\Phi_{\mathrm{T}_{\ell, z}}$ and $\Phi_{\mathrm{F}_{\ell, z}}$. These are equal to the injected quantity $y_{z}$, if the line is fully within the sub-graph, or 0 , otherwise. This condition is modelled with the scalar products $\boldsymbol{H}_{\mathrm{F}_{(\ell, \cdot)}} \boldsymbol{x}_{(\cdot, z)}$ and $\boldsymbol{H}_{\mathrm{T}_{(\ell, \cdot)}} \boldsymbol{x}_{(\cdot, z)}$, whose values are either 1 , if the "from" or "to" node of line $\ell$ is included in zone $z$, or 0 , if not. Therefore, the flow of units is prevented, unless both scalar products in (12)-(13) are equal to 1 . In this case, $\varphi_{\ell, z}$ is limited by $y_{z}$, which always represents an upper bound to the highest possible flow of units.

To summarize, the grid partitioning requires the set of decision variables $\Xi_{\mathrm{G}}=\left\{x_{n, z}, c_{n, z}, \forall n, \forall z ; y_{z}, \forall z ; \varphi_{\ell, z}, \forall \ell, \forall z\right\}$ constrained by (5) - (13) in the upper-level problem of the proposed bilevel methodology. Section IV of the paper describes in detail the linearization of the bilinear terms that appear in constraints (7), (12) and (13) using the Big-M approach [27].

\section{B. Dynamic Reserve Procurement}

The proposed methodology allows the grid operator to identify and clear $\mathcal{Z}$ independent reserve markets, each of them corresponding to a zone of the partition. Although the objective remains to minimize the total expected system cost, zonal requirements can be differentiated while respecting the merit order of generators that participate in each reserve market. The dynamic reserve procurement model constitutes one of the two lower-level problems in the bilevel structure of the proposed methodology and it is formulated as 


$$
\begin{array}{ll}
\min _{\Xi_{\mathrm{R} z}} \mathcal{C}_{\mathrm{R}, z}=\sum_{g \in \mathcal{G}}\left(C_{g}^{+} r_{g}^{+}+C_{g}^{-} r_{g}^{-}\right) & \\
\text {s.t. } & \forall z \in \mathcal{Z}, \\
\sum_{g \in \mathcal{G}} r_{g, z}^{+} \geq \lambda_{z}^{+}, & \forall z \in \mathcal{Z}, \\
\sum_{g \in \mathcal{G}} r_{g, z}^{-} \geq \lambda_{z}^{-}, & \forall z \in \mathcal{Z}, \quad \forall g \in \mathcal{G}, \\
0 \leq r_{g, z}^{+} \leq R_{g}^{+}\left(\boldsymbol{H}_{\mathrm{G}_{(g, \cdot)}} \boldsymbol{x}_{(\cdot, z)}\right), & \forall z \in \mathcal{Z}, \quad \forall g \in \mathcal{G}, \\
0 \leq r_{g, z}^{-} \leq R_{g}^{-}\left(\boldsymbol{H}_{\mathrm{G}_{(g, \cdot)}} \boldsymbol{x}_{(\cdot, z)}\right), & \forall g \in \mathcal{G}, \\
r_{g}^{+}=\sum_{z \in \mathcal{Z}} r_{g, z}^{+}, & \forall g \in \mathcal{G} \\
r_{g}^{-}=\sum_{z \in \mathcal{Z}} r_{g, z}^{-}, &
\end{array}
$$

where $\Xi_{\mathrm{R}_{z}}=\left\{r_{g, z}^{+}, r_{g, z}^{-}, \forall g, \forall z\right\}$ is the set of decision variables: $r_{g, z}^{+}$and $r_{g, z}^{-}$represent up- and downward reserve from generator $g$ in zone $z$, respectively. Note that since $x_{n, z}$ is an upper-level variable, it enters (14) as a parameter, thus rendering model (14) a linear programming problem. This structure allows to use the associated Karush-Kuhn-Tucker (KKT) conditions to reformulate the bilevel structure into a mathematical problem with equilibrium constraints (MPEC). The upward and downward zonal requirements $\lambda_{z}^{+}$and $\lambda_{z}^{-}$ imposed through (14b)-(14c) also enter this formulation as parameters, since they are upper-level decision variables. Zonal reserve requirements $\lambda_{z}$ are fulfilled by generators that belong to the corresponding zones. The scalar product in (14d)-(14e) between $\boldsymbol{H}_{\mathrm{G}_{(g, \cdot)}}$ and $\boldsymbol{x}_{(\cdot, z)}$ indicates whether generator $g$ is eligible for providing reserve to zone $z$. Finally, (14f)-(14g) define the overall reserve to be acquired from each generator.

\section{Transmission Capacity Allocation}

In cases when flexible resources are concentrated in a certain zone of the system, the grid operator could set aside part of the cross-zonal transmission capacity in order to facilitate the exchange of reserves. This aspect is modelled in the proposed formulation by means of an additional set of upperlevel decision variables $\Xi_{\mathrm{C}}=\left\{h_{\ell, z}, \Gamma_{\ell, z}, \Gamma_{\ell}\right\}$, which defines the available capacity for energy trading on each cross-zonal line. Consider Fig. 1 where a 4-bus system is partitioned in two possible configurations. Note that as the zones are defined

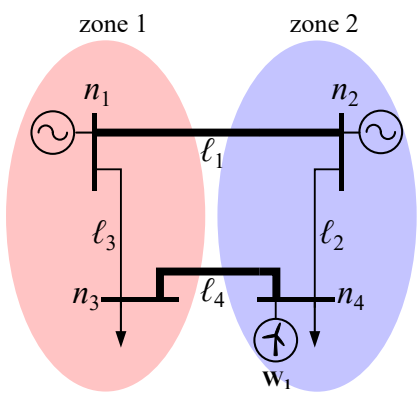

(a)

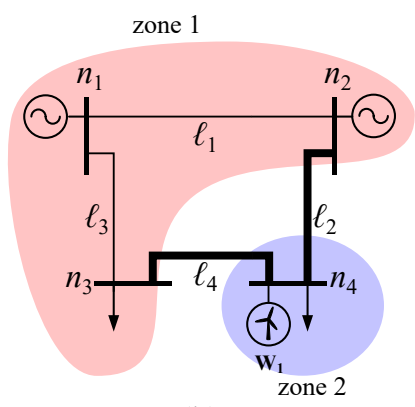

(b)
Fig. 1. Illustrative case of two possible configurations of grid partitioning on a 4-bus system. Thicker lines indicates cross-zonal interconnections. dynamically, so are the cross-zonal lines eligible for reserve exchange, i.e., lines $\ell_{1}$ and $\ell_{4}$ in Fig. 1(a) as opposed to lines $\ell_{2}$ and $\ell_{4}$ in Fig. 1(b). Therefore, the partitioning identifies endogenously the lines whose capacity can be set aside for reserve exchange. This aspect is modelled with an auxiliary integer variable $h_{\ell, z}$, defined as the number of nodes that a line $\ell$ has in zone $z$, according to the following expression

$$
h_{\ell, z}=\boldsymbol{H}_{\mathrm{F}_{(\ell, \cdot)}} \boldsymbol{x}_{(\cdot, z)}+\boldsymbol{H}_{\mathrm{T}_{(\ell, \cdot)}} \boldsymbol{x}_{(\cdot, z)}, \quad \forall \ell \in \mathcal{L}, \forall z \in \mathcal{Z} .
$$

The values that $h_{\ell, z}$ can take are: 0,1 or 2 and they reflect all possible configurations between lines and zones. In the first case $h_{\ell, z}=0$, the line is totally outside the considered zone, e.g., line $\ell_{2}$ with respect to zone 1 in Fig. 1(a); in the second case $h_{\ell, z}=1$, the line is cross-zonal because only one of the two nodes is included in a zone, e.g., line $\ell_{1}$ in Fig. 1(a); in the third case, $h_{\ell, z}=2$ indicates a line that is fully included in the considered zone, e.g., $\ell_{1}$ in Fig. 1(b). Only when $h_{\ell, z}=1$ a portion of the capacity of line $\ell$ is set aside, while the other cases identify domestic lines whose capacity is entirely allocated for energy trading in the day-ahead market. The following set of constraints limits the capacity allocation for reserve exchange $\Gamma_{\ell, z}$ to or from zone $z$ on line $\ell$,

$$
\begin{aligned}
& \Gamma_{\ell, z} \leq \chi F_{\ell} h_{\ell, z}, \quad \forall \ell \in \mathcal{L}, \quad \forall z \in \mathcal{Z}, \\
& \Gamma_{\ell, z} \leq \chi F_{\ell}\left(2-h_{\ell, z}\right), \quad \forall \ell \in \mathcal{L}, \quad \forall z \in \mathcal{Z},
\end{aligned}
$$

where a predefined parameter $\chi$ is included in order to limit the maximum capacity that can be withdrawn from day-ahead market and $F_{\ell}$ indicates the line rating. When $h_{\ell, z}$ is either 0 or 2 , one of the above constraints binds $\Gamma_{\ell, z}$ to be zero, thus preventing any capacity of that line to be set aside. In the remaining case, $h_{\ell, z}=1$, both (16) and (17) state that the share of capacity can be up to the $\chi \%$ of the line rating. The remaining constraints include

$$
\begin{array}{lll}
0 \leq \Gamma_{\ell, z} \leq \Gamma_{\ell}, & \forall \ell \in \mathcal{L}, \quad \forall z \in \mathcal{Z}, \\
\Gamma_{\ell}=\frac{1}{2} \sum_{z} \Gamma_{\ell, z}, & \forall \ell \in \mathcal{L}, &
\end{array}
$$

that serve a twofold purpose. The first is to enforce nonnegativity of $\Gamma_{\ell, z}$, the second is to define $\Gamma_{\ell}$, which is used to define uniquely the value of capacity to be set aside on each line $\ell$, regardless of the zone considered. Note how the use of $\frac{1}{2}$ prevents counting the line capacity twice in (19).

Using the above transmission capacity allocation scheme, day-ahead power flows are bounded by $F_{\ell}-\Gamma_{\ell}$, instead of $F_{\ell}$ as in the conventional day-ahead market of the sequential approach. This limits the expected power flows at the dayahead stage, in order to ensure that enough transmission capacity is available during real-time operation.

\section{Zonal Preemptive Problem Formulation}

The proposed methodology builds upon the recent work in [10] and [11] that also adopts a stochastic bilevel framework for setting reserve requirements. While previous studies considered either a single zone or a predefined zonal setup, we improve the positioning of reserves by defining zone boundaries together with their reserve requirements. The complete 
problem formulation, where both reserve and transmission capacity are dynamically allocated, is formulated as

$$
\begin{aligned}
& \min _{\Xi_{\mathrm{M} z}} \mathcal{C}_{\mathrm{M}_{z}}=\mathcal{C}_{\mathrm{R}}+\mathcal{C}_{\mathrm{D}}+\sum_{s \in \mathcal{S}} \pi_{s} \mathcal{C}_{\mathrm{B}, s} \\
& \text { s.t. } \quad\left(r_{g}^{+}, r_{g}^{-}\right) \in \arg \left\{\begin{array}{l}
\text { minimize } \mathcal{C}_{R_{z}} \\
\Xi_{\mathrm{R}_{z}}^{\prime} \\
\text { subject to } \\
\text { constraints }(14 \mathrm{~b})-(14 \mathrm{~g})
\end{array}\right\} \text {, } \\
& \left(p_{g}, w_{j}\right) \in \arg \left\{\begin{array}{l}
\underset{\Xi_{D}^{\prime}}{\operatorname{minimize}} \mathcal{C}_{\mathrm{D}} \\
\text { subject to } \\
\text { constraints }(2 \mathrm{~b})-(2 \mathrm{f})
\end{array}\right\}, \\
& \lambda_{z}^{+} \geq 0, \quad \lambda_{z}^{-} \geq 0, \\
& \text { (3b) - (3h), Balancing market, } \forall s \in \mathcal{S} \text {, } \\
& \text { (5) - (13), Grid partitioning, } \\
& \text { (15) - (19), Capacity allocation, }
\end{aligned}
$$$$
\forall z \in \mathcal{Z},(20 \mathrm{~d})
$$

where $\Xi_{\mathrm{M}_{z}}=\left\{\lambda_{z}^{+}, \lambda_{z}^{-}, \forall z \cup \Xi_{\mathrm{R}} \cup \Xi_{\mathrm{D}} \cup \Xi_{\mathrm{B}, \mathrm{s}}, \forall s \cup \Xi_{\mathrm{G}} \cup \Xi_{\mathrm{C}}\right\}$ is the set of upper-level decision variables. This comprises: zonal reserve requirements $\lambda_{z}$; reserve, day-ahead and balancing market decision variables, which are constrained by the corresponding lower-level problems; grid partitioning and transmission capacity allocation variables $\Xi_{\mathrm{G}}$ and $\Xi_{\mathrm{C}}$, respectively. Lower-level problem (20b) accounts for the dynamic reserve allocation strategy described in III-B, whereas (20c) is the same day-ahead market clearing model as in model (2) where line ratings $F_{\ell}$ are substituted with $\left(F_{\ell}-\Gamma_{\ell}\right)$.

The mathematical structure of model (20), ensures that the reserve capacity and day-ahead energy markets are cleared independently, while none of these markets can foresee the future re-dispatch actions. This modelling approach allows to replicate the European market architecture and in the meantime the upper-level problem can still anticipate the effect of reserve market parameters, i.e. zonal setting, inter-area transmission allocation and reserve requirements, on every market and on the total expected system cost. In particular, the grid partitioning constraints embedded in the upper-level problem, enable to define the optimal reserve zone configuration such that the total expected system cost is minimized.

For the sake of clarity, Fig. 2 illustrates the structure and the different components of the Zonal Preemptive model.

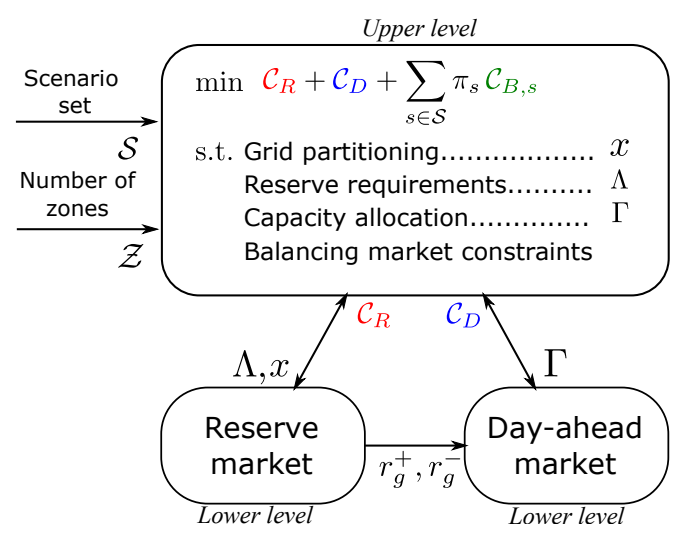

Fig. 2. Conceptual representation of the proposed methodology.
This structure establishes a closed-loop feedback between the upper-level problem, defining the optimal reserve market parameters (i.e. reserve zones $x$, reserve requirements $\Lambda$ and transmission capacity allocation $\Gamma$ ) and the lower-level problems, accounting for their impact on the day-ahead and balancing markets. According to this structure, the reserve zones defined by the upper-level problem are fed as fixed parameters in the reserve capacity market. Then, the procured reserved quantities are transmitted to the day-ahead and balancing markets that are cleared for every uncertainty realization $s \in \mathcal{S}$. As opposed to the existing sequential approach, in which all reserve market parameters are exogenously defined, the Zonal Preemptive model intrinsically optimizes these values in order to minimize the total expected system cost.

Despite that our Zonal Preemptive model shares the same objective function and constraints as the stochastic energy and reserve co-optimization model presented in Section II-B, our formulation guarantees that the reserve capacity and dayahead energy markets respect the merit-order principle, such that the cost recovery and revenue adequacy properties hold for any uncertainty realization. The previous works in [10] and [11] were aiming to improve the efficiency of the reserve procurement process by tuning the zonal reserve requirements and inter-zonal transmission capacity allocation that allows the exchange of reserve products. However, both models still require the ex-ante definition of reserve zones that is a source of inefficiency if energy market reaction and network congestions are not taken into consideration during the decision-making process. To this end, our Zonal Preemptive model comes to fix precisely this design flaw of the previous models by accounting for an additional degree of freedom, i.e., the definition of reserve zones, accounting for the structure of forecast errors, the impact of reserve market setting on the energy trading and the deliverability of reserves in real-time operation.

\section{SOLUTION APPROACH}

All bilinear terms that appear in the grid partitioning constraints can be expressed as a product between a binary and an integer variable, i.e., $y_{z}\left(\boldsymbol{H}_{\mathrm{F}_{(\ell, .)}} \boldsymbol{x}_{(\cdot, z)}\right), y_{z}\left(\boldsymbol{H}_{\mathrm{T}_{(\ell, .)}} \boldsymbol{x}_{(\cdot, z)}\right)$ and $c_{n, z} y_{z}$. The linearization of these terms is illustrated for the latter case, by introducing an auxiliary variable $u_{n, z}$ that replaces the product $c_{n, z} y_{z}$ in (7) according to the Big-M approach [27]. The following constraints are added

$$
\begin{aligned}
y_{z}-M\left(1-c_{n, z}\right) & \leq u_{n, z} \leq y_{z}-m\left(1-c_{n, z}\right) \\
m \cdot c_{n, z} & \leq u_{n, z} \leq M \cdot c_{n, z}
\end{aligned}
$$

where $m=1$ and $M=\mathcal{N}$, i.e., the number of nodes in the system. Note that the specific values of $m$ and $M$ are used for all the bilinear terms that appear in the grid partitioning constraints. These values are straightforward to derive: each bilinear term is either 0 or equal to the sub-graph cardinality $y_{z}$, thus 1 and $N$ always represent valid bounds.

For any feasible partition of the system defined by the upper level variables, each lower level problem is linear and convex. Thus, the bilevel problem is reformulated as an MPEC, where each lower-level problem is replaced by the corresponding KKT conditions. This step introduces additional auxiliary 
binary variables in order to linearize the complementarity slackness constraints in the KKT conditions. The MPEC problem is then recast as a single-level MILP by using the Big$\mathrm{M}$ method. This solution approach is typically used in power systems research, although authors of [28] recently pointed out some critical limitations.

As already mentioned, we envision the utilization of the proposed Zonal Preemptive model mainly as decision-support tool for the network operators rather than as an online marketclearing algorithm. Hence, the computational requirements regarding the maximum solution time of the model are not strict. Nevertheless, to reduce the computational burden of the proposed model, we take advantage of the special structure of the bilevel problem, whose second-stage constraints are independent per scenario, and we implement a multicut Bender's decomposition scheme [29]. The complete set of KKT conditions of the lower-level problems, along with the formulations of the Bender's master problem and subproblems, are provided in Appendix A and Appendix B, respectively.

\section{RESUlts}

\section{A. Wind Power Scenarios}

In this paper, probabilistic forecast errors of wind power generation are assumed to follow a Beta distribution, whose parameters are calculated according to [30] and the error variance follows a quadratic function of the per unit point forecast. The spatial correlation structure in wind power generation at different locations is modelled by means of a Gaussian copula function with a rank correlation matrix based on actual wind power realizations from the Danish system [31]. A large number of scenarios is then generated by sampling the resulting multivariate joint probabilistic forecast for a single time-period. In order to keep computational tractability in the stochastic programs, scenario sets $\mathcal{S}_{i}$ are reduced accordingly to 100 realizations using the fast-forward scenario reduction technique [32]. The number of 100 realizations has been chosen on the basis of an in-sample stability analysis, where for each tested set size, the proposed methodology has been simulated 10 times, in order to observe the variability of the objective function in terms of the maximum pairwise difference between different runs. Table I lists the results of the in-sample stability analysis for the RTS24 system and it shows that increasing the set size beyond 100 does not show

TABLE I

MAXIMUM OBJECTIVE FUNCTION PAIRWISE DIFFERENCE. VALUES IN PERCENTAGE OF THE MEAN VALUE.

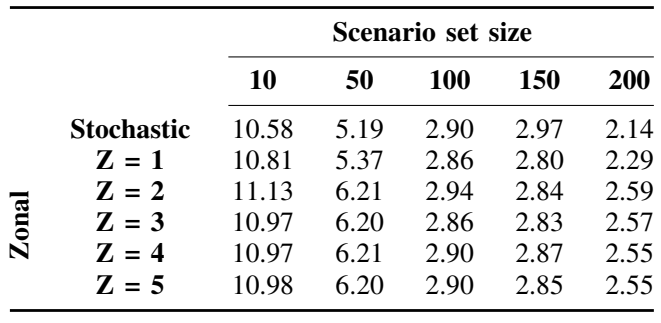

considerable improvement compared against the increased computational time.

\section{B. Stability Analysis (IEEE RTS-24 System)}

The proposed methodology is showcased on a modified version of the IEEE RTS-24 system, whose detailed parameters are available in [33]. In particular, three lines are de-rated and six wind farms with $200 \mathrm{MV}$ installed capacity are included in the system at selected locations. Simulation results are benchmarked against the stochastic co-optimization of energy and reserve (4) and the conventional approach of sequentially cleared markets, i.e., (1), (2) and (3). Reserve requirements $\Lambda$ in model (1) are calculated as

$$
\begin{aligned}
& \Lambda^{+}=\widehat{W}_{\mathrm{tot}}-\widehat{\boldsymbol{F}}_{W}^{-1}(q) \\
& \Lambda^{-}=\widehat{\boldsymbol{F}}_{W}^{-1}(1-q)-\widehat{W}_{\mathrm{tot}}
\end{aligned}
$$

where $\widehat{W}_{\text {tot }}$ and $\widehat{\boldsymbol{F}}_{W}$ represent the expected value and the predictive Cumulative Density Function (CDF) of the total wind power probabilistic forecast, respectively, while the predetermined quantile $q$ of the distribution is chosen in line with grid operator's risk aversion. The proposed methodology is tested either with or without transmission capacity allocation, considering three values of the parameter $\chi$ and a minimal zonal size of 4 nodes.

In order to test the stability of the considered models against small deviations in the uncertain wind power generation, upand downward reserve levels obtained with scenario set $\mathcal{S}_{1}$ are plugged into (2) and (3), where the uncertainty is described by 10 different scenario sets $\mathcal{S}_{i}(i=2, \ldots, 11)$, based on the same multivariate probabilistic forecast. Figure 3 shows the corresponding total cost, which are normalized with the solution of the stochastic model obtained with each set $\mathcal{S}_{i}$. It stands out that the zonal approach outperforms the sequential one both in terms of stability and cost effectiveness, regardless of the chosen quantile $q$ for setting reserve levels. The zonal model shows an improvement with just 2 zones, whereas it coincides with model [10] if a single zone is considered.

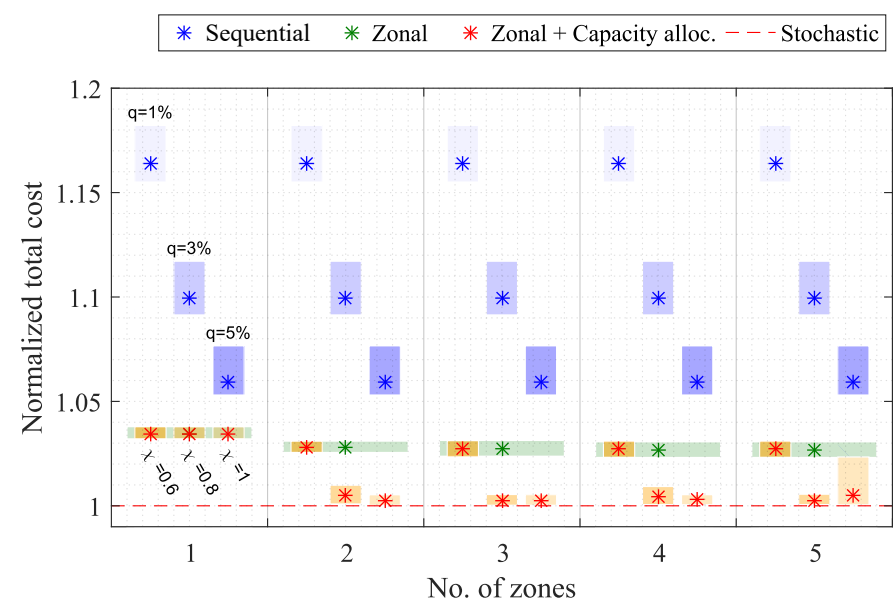

Fig. 3. Stability analysis in the RTS 24 bus system. Asterisks represent the mean values, upper and lower edges of rectangles represent max and min values, respectively. 
TABLE II

COST BREAKDOWN OF SELECTED MODELS

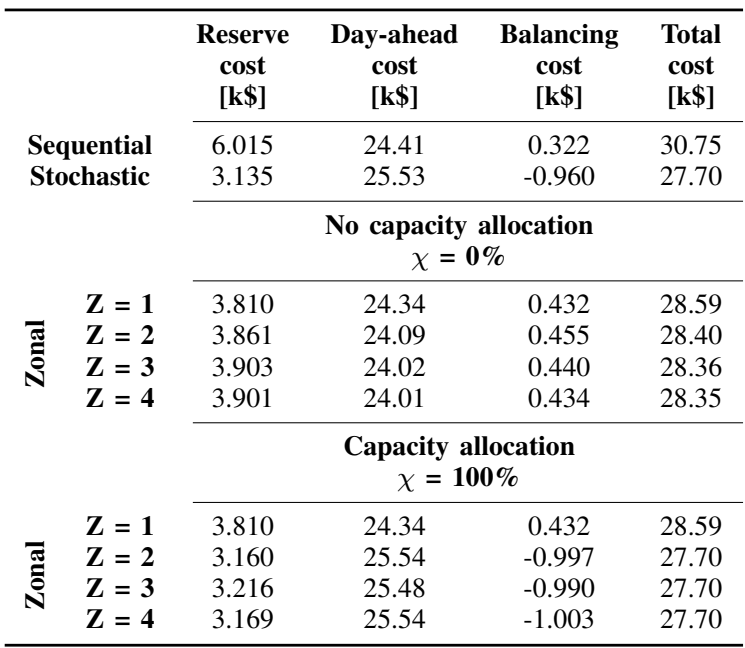

The effect of allowing transmission capacity allocation on cross-zonal lines is to lower the costs further, provided that more than $60 \%$ of eligible line capacities is withdrawn from the day-ahead market. This result resembles the line-switching approach, where the cost effectiveness of a dispatch can be improved if power flows are re-routed by switching off selected lines. In this case, reducing the capacity allocated to the day-ahead market ensures that enough headroom is available for balancing the system, thus avoiding bottlenecks that would result in expensive corrective actions.

\section{Cost Breakdown}

Table II shows the cost breakdown of selected models solved with the same scenario set $\mathcal{S}_{1}$. The conventional model in this case relies on the top and bottom $3 \%$ of the total wind power distribution for setting reserve requirements. This approach results in higher cost for reserves, as it cannot account neither for their location in the system nor for the network constraints that might hinder their accessibility. Instead, the preemptive model with a single zone is able to regulate reserve requirements based on expected re-dispatch actions. Although improving the results considerably, this approach still relies on a single reserve market and thus it follows the merit order of generators' reserve capacity offers. The implication is that while total reserve levels can be fine-tuned, their location and position in the system cannot.

This aspect motivates the introduction of a zonal setup that provides the grid operator with additional flexibility to approximate the ideal solution. As the number of zones increases, so does the ability to lower the costs towards the stochastic model and to optimally allocate reserves. Note that the partitioning in the proposed methodology is solely driven by the total expected costs and it does not require any root node to be pre-specified, which could introduce a degree of arbitrariness in the partition. Therefore, it inherently considers the availability of reserves in the system, their procurement and their activation costs given the network limitations. Table III summarizes the zonal reserve costs referring to the case of 3
TABLE III

ZONAL RESERVE COST OF GRID PARTITIONS IN FIG. 4

\begin{tabular}{|c|c|c|c|c|c|c|c|c|}
\hline & \multicolumn{4}{|c|}{$\begin{array}{c}\text { (a) No capacity allocation } \\
\chi=0 \%\end{array}$} & \multicolumn{4}{|c|}{$\begin{array}{c}\text { (b) Capacity allocation } \\
\chi=100 \%\end{array}$} \\
\hline & \multicolumn{3}{|c|}{$\begin{array}{c}\text { Reserve volume } \\
{[\mathrm{MW}]}\end{array}$} & \multirow[t]{2}{*}{$\begin{array}{l}\text { Avg. } \\
\text { cost } \\
{[\$ / \mathrm{MW}]}\end{array}$} & \multicolumn{3}{|c|}{$\begin{array}{c}\text { Reserve volume } \\
\quad[\mathrm{MW}]\end{array}$} & \multirow[t]{2}{*}{$\begin{array}{c}\text { Avg. } \\
\text { cost } \\
{[\$ / \mathrm{MW}]}\end{array}$} \\
\hline & $U p$ & $D w$ & Total & & $U p$ & $D w$ & Total & \\
\hline Zone 1 & 60.0 & 35.7 & 95.7 & 15 & 0 & 67.9 & 67.9 & 8.54 \\
\hline Zone 2 & 75.9 & 112.1 & 188.0 & 12 & 27.5 & 143.9 & 171.4 & 9.72 \\
\hline Zone 3 & 40.0 & 0 & 40.0 & 14.3 & 30.0 & 30.0 & 60.0 & 13.33 \\
\hline Total & 175.9 & 147.8 & 323.7 & 12.7 & 57.5 & 241.8 & 299.3 & 10.23 \\
\hline
\end{tabular}

(a)

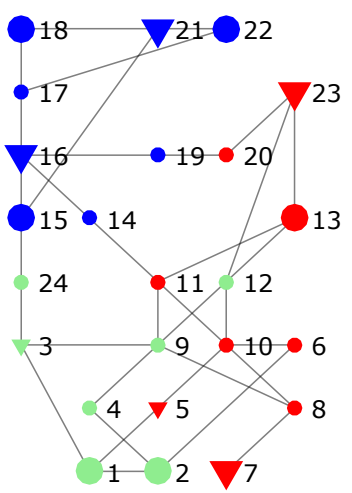

(b)

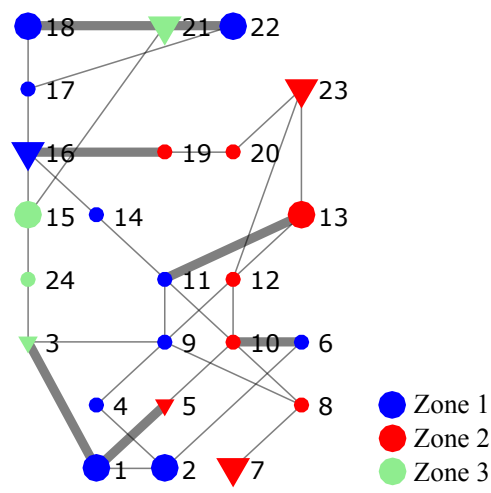

Fig. 4. Partition of the RTS-24 system into 3 zones without (a) and with (b) transmission capacity allocation on selected lines. Triangles indicate wind power, large markers indicate the presence of generators.

zones in Fig. 4. The zonal setup allows to procure nearly $60 \%$ of total requirements from zone 2, where the cost per MW is lower. Zone 3 instead procures less reserve, but from more expensive generators ensuring that enough balancing power is located close to wind farms at nodes 3 and 5 .

Two effects are evident as we allow for transmission capacity allocation on cross-zonal lines: the first is that reserve costs decrease, since more power can be reserved from cheaper generators, while ensuring that they can deliver it to the grid; the second is that day-ahead costs increase, as we reduce the network capacity available for energy trading. A consequence of this latter aspect is that less wind power will be dispatched at this stage. To compensate for that and to avoid expensive wind curtailment penalties, more downward reserve needs to be procured and activated, as can be seen from Table III.

\section{RTS-96 Case Study}

The proposed methodology is also tested in the 3-area RTS96 system, for which relevant data is taken from [34]. The system is considered during the peak hour with a total demand of $7.5 \mathrm{GW}, 18 \%$ of which is covered by wind power located in 5 locations. Original line ratings are used, whereas the minimum power output of controllable units is set to zero. In order to exclude the generator-specific resolution of the stochastic approach, a minimal zonal size of 10 nodes has been enforced in this system. 
Figure 5 shows the resulting total expected costs, where we compare the proposed methodology to the sequential approach with varying reserve requirements (i.e. corresponding to different quantiles $q$ in (24) and (23)) and a zonal model with a predetermined partitioning variable $x_{n, z}$, according to the standard partitioning of this system into three zones. This approach allows us to isolate the contribution of the flexible zone boundaries definition provided by the proposed Zonal Preemptive approach. The partition into 3 zones is shown in Fig. 6 together with the common subdivision of the RTS96 system into 3 areas. The total expected costs indicate that tuning reserve requirements while considering a single reserve market, i.e., $Z=1$, does not result in significant savings, as opposed to the sequential approach. The Zonal Preemptive approach with a fixed partition that adheres to the 3 areas in Fig. 6 corresponds the methodology in [10] and it performs better than the single zone. However, as the zones are dynamically determined by the partitioning variables $x_{n, z}$, the total costs fall near the lower bound represented by the stochastic co-optimization of energy and reserve. It suffices to split the system into 2 zones to stay within the $0.1 \%$ increase from the lower bound, even without allocating transmission capacity on cross-zonal lines. The resulting large-scale MILP problem is solved with Gurobi setting a $0.1 \%$ optimality gap on a quad-core laptop with $8 \mathrm{~GB}$ of RAM and $2.4 \mathrm{GHz}$ of CPU. Bender's decomposition converged in $3.50 \cdot 10^{2} \mathrm{~s}$ with 1 zone, $2.15 \cdot 10^{3} \mathrm{~s}$ with 2 zones and $4.04 \cdot 10^{4} \mathrm{~s}$ with 3 zones.

\section{E. Policy implications for reserve zones configuration}

As discussed in [23] and emphasized forcefully in Article 37 of [35], European system operators are setting the ground for increased cross-border cooperation. Tasks for regional coordination centers include regional sizing of reserve capacity and the facilitation of regional procurement of balancing services. However, as it is shown in our case studies, simply enlarging the pool of reserves might not be sufficient to improve overall market efficiency, if network constraints are not taken into account. From a policy perspective, the results from the RTS96 system, point to the fact that as reserve and balancing markets move from national to regional scale in order to pool more flexible resources and mitigate risks, the need for redefining zonal boundaries becomes increasingly important. To this end, our methodology clearly indicates the need for 'smart' splitting of reserve capacity markets into a number of zones, in order to enable the optimal allocation of generation resources between energy and reserve services. In addition, our case study shows how the ideal lower bound can be approximated with a dynamic definition of zones, as opposed to a single large zone or smaller predefined zones that obey conventional boundaries such as national borders, geographical location or ownership.

As previously explained in Section IV, we do not advocate for the adoption of the proposed methodology as an online market-clearing mechanism, but rather as decision-support tool for different stages of operational planning, e.g., on a daily, weekly or seasonal basis. In this case, the frequency of updating the reserve zones configuration should take into

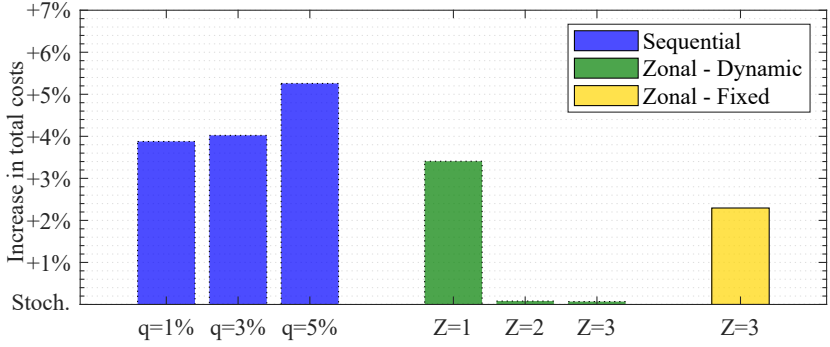

Fig. 5. Increase in total expected costs for the load demand peak hour in the RTS-96 system. Costs normalized with the stochastic solution.

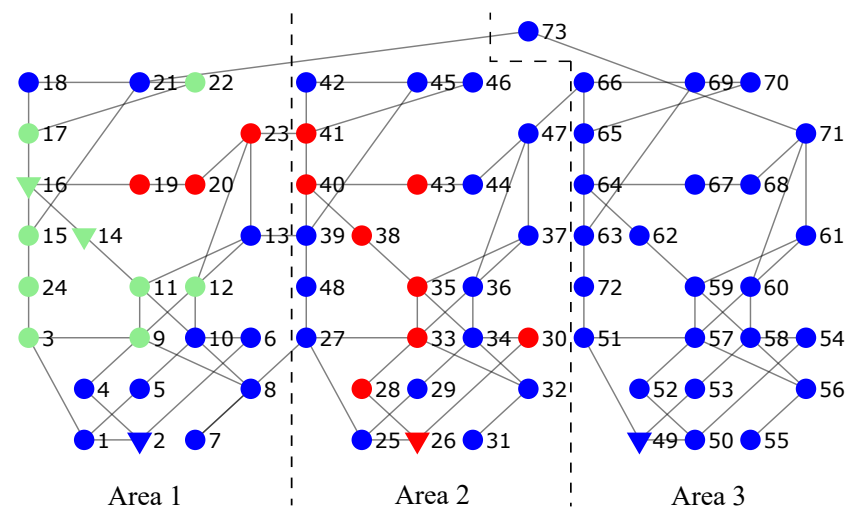

Fig. 6. Partition of the 3-area IEEE RTS-96 system according to the Zonal Preemptive model with $\chi=0$. Triangles indicate wind farms.

consideration operators' requirements but also facilitate the operations of the various stakeholders who participate in the electricity markets. On the one hand, updating the zone boundaries on a daily basis should yield the highest cost reductions, since the process would benefit from the most recent information and day-ahead forecasts. The downside of a frequent redefinition of zones, however, would be increased uncertainty and volatility for those flexible units that provide the balancing services, as they might be frequently re-assigned to different zones. On the other hand, a seasonal partition of the system could mitigate the organizational burden for market participants, but it would unavoidably rely on a more coarse description of the system and lower quality forecasts that will undermine a portion of the potential cost savings.

Considering the above discussion, it becomes apparent that the proposed methodology provides the operators with the mathematical tools to perform a dynamic configuration of the zonal reserve setup. However, the frequency of this zonal reconfiguration involves also some regulatory consideration in order to ensure a fair compromise between the preferences of the system operator and the market participants.

\section{CONCLUSION}

This paper described a novel methodology for reserve procurement that further approximates the efficiency of the stochastic co-optimization of energy and reserves in terms of total operating costs, while still respecting the existing market principles. Building upon recent work on stochastic bilevel optimization, we embed grid partitioning constraints in the upper-level problem and use them to determine not only the zonal reserve requirements but the zonal boundaries as well. 
Unlike other partitioning schemes, our methodology is solely driven by the total expected system costs and the most recent uncertainty forecasts, instead of relying on historical data that may not reflect the actual system state. The proposed model allows grid operators to perform a dynamic zoning of the system for reserve procurement, depending upon generation uncertainty and network limitations. In addition, this Zonal Preemptive model can contribute to the ongoing policy discussion towards a common European reserve capacity market, where reserve zones are dynamically defined upon system conditions instead of geographical borders. Simulation results on the RTS96 system show that the stochastic lower bound can be adequately approximated within the $0.1 \%$ with only two zones, even if a minimal zonal size of 10 nodes is required. This result suggests that the computational burden of the proposed approach can be reduced by limiting the number of reserve zones, without a major efficiency loss in terms of expected system cost. Moreover, the combination of dynamic reserve procurement with cross-zonal transmission capacity allocation has shown to be beneficial in highly congested systems. Setting aside part of the available transmission capacity grants the grid operator additional flexibility to approach the efficiency of the stochastic dispatch.

Future work will address the current limitations of the proposed methodology considering inter-temporal constraints, which may affect the partitioning of the system and the deployment of reserves. Extending the proposed methodology to a multi-period formulation would further improve the positioning of flexible generation capacity in the system, as it would allow a more accurate representation of power system dynamics. Ramping rates of generators as well as energy storage devices may significantly affect the definition of zones in the system and the corresponding reserve requirements.

\section{APPENDIX A}

This section presents the KKT conditions of the dynamic reserve procurement problem in Section III-B and the day-ahead market clearing in Section II. The corresponding optimization problems are repeated in a standard notation to ease the identification of dual variables, which are indicated as $\gamma^{*}$ for constraint $(*)$. Lastly, symbol $\perp$ indicates the complementarity conditions between the constraints.

\section{A. Zonal reserve market}

1) Problem formulation:

$\min _{\Xi_{\mathrm{R}_{z}}} \mathcal{C}_{\mathrm{R}, z}=\sum_{g \in \mathcal{G}}\left(C_{g}^{+} r_{g}^{+}+C_{g}^{-} r_{g}^{-}\right)$

s.t.

$\lambda_{z}^{+}-\sum_{g \in \mathcal{G}} r_{g, z}^{+} \leq 0, \quad \forall z \in \mathcal{Z}$,

$\lambda_{z}^{-}-\sum_{g \in \mathcal{G}} r_{g, z}^{-} \leq 0, \quad \forall z \in \mathcal{Z}$,

$r_{g, z}^{+}-R_{g}^{+}\left(\boldsymbol{H}_{\mathrm{G}_{(g, \cdot)}} \boldsymbol{x}_{(\cdot, z)}\right) \leq 0, \quad \forall z \in \mathcal{Z}, \quad \forall g \in \mathcal{G}$,

$r_{g, z}^{-}-R_{g}^{-}\left(\boldsymbol{H}_{\mathrm{G}_{(g, \cdot)}} \boldsymbol{x}_{(\cdot, z)}\right) \leq 0, \quad \forall z \in \mathcal{Z}, \quad \forall g \in \mathcal{G}$,

$-r_{g, z}^{+} \leq 0$

$\forall z \in \mathcal{Z}, \quad \forall g \in \mathcal{G}$,

$$
\begin{array}{ll}
-r_{g, z}^{-} \leq 0 & \forall z \in \mathcal{Z}, \quad \forall g \in \mathcal{G}, \\
r_{g}^{+}-\sum_{z \in \mathcal{Z}} r_{g, z}^{+}=0, & \forall g \in \mathcal{G}, \\
r_{g}^{-}-\sum_{z \in \mathcal{Z}} r_{g, z}^{-}=0, & \forall g \in \mathcal{G}
\end{array}
$$

2) KKT conditions:

$C_{g}^{+}-\gamma_{z}^{(25 \mathrm{~b})}+\gamma_{g, z}^{(25 \mathrm{~d})}-\gamma_{g, z}^{(25 \mathrm{f})}+\gamma_{g}^{(25 \mathrm{~h})}=0, \quad \forall g, \forall z$,

$C_{g}^{-}-\gamma_{z}^{(25 \mathrm{c})}+\gamma_{g, z}^{(25 \mathrm{e})}-\gamma_{g, z}^{(25 \mathrm{~g})}+\gamma_{g}^{(25 \mathrm{i})}=0, \quad \forall g, \forall z$,

$0 \geq \lambda_{z}^{+}-\sum_{g \in \mathcal{G}} r_{g, z}^{+} \quad \perp \quad \gamma_{z}^{(25 \mathrm{~b})} \geq 0$,

$\forall z$

$0 \geq \lambda_{z}^{-}-\sum_{g \in \mathcal{G}} r_{g, z}^{-} \quad \perp \quad \gamma_{z}^{(25 \mathrm{c})} \geq 0, \quad \forall z$

$0 \geq r_{g, z}^{+}-R_{g}^{+}\left(\boldsymbol{H}_{\mathrm{G}_{(g, \cdot)}} \boldsymbol{x}_{(\cdot, z)}\right) \perp \gamma_{g, z}^{(25 \mathrm{~d})} \geq 0$,

$\forall g, \forall z$

$0 \geq r_{g, z}^{-}-R_{g}^{-}\left(\boldsymbol{H}_{\mathrm{G}_{(g, .)}} \boldsymbol{x}_{(\cdot, z)}\right) \perp \gamma_{g, z}^{(25 \mathrm{e})} \geq 0, \quad \forall g, \forall z$

$0 \geq-r_{g, z}^{+} \perp \gamma_{g, z}^{(25 f)} \geq 0$,

$\forall g, \forall z$

$0 \geq-r_{g, z}^{-} \perp \quad \gamma_{g, z}^{(25 \mathrm{~g})} \geq 0$,

$\forall g, \forall z$

$r_{g}^{+}-\sum_{z \in \mathcal{Z}} r_{g, z}^{+}=0$,

$\forall g$

$r_{g}^{-}-\sum_{z \in \mathcal{Z}} r_{g, z}^{-}=0$

$\forall g$.

\section{B. Day-ahead market}

1) Problem formulation:

$\min _{\Xi_{\mathrm{D}}} \mathcal{C}_{\mathrm{D}}=\sum_{g \in \mathcal{G}} C_{g} p_{g}$

s.t.

$\sum_{g \in \mathcal{G}} p_{g}+\sum_{j \in \mathcal{J}} w_{j}-\sum_{n \in \mathcal{N}} D_{n}=0$,

$\underline{P}_{g}+r_{g}^{-, *}-p_{g} \leq 0$

$\forall g \in \mathcal{G}, \quad$ (36c)

$p_{g}-\bar{P}_{g}+r_{g}^{+, *} \leq 0$,

$\forall g \in \mathcal{G}$,

$-w_{j} \leq 0$,

$\forall j \in \mathcal{J}$,

$w_{j}-\widehat{W}_{j} \leq 0$,

$\forall j \in \mathcal{J}$,

$\boldsymbol{M}_{(\ell, \cdot)}\left(\boldsymbol{H}_{\mathrm{G}}^{\top} \boldsymbol{p}+\boldsymbol{H}_{\mathrm{J}}^{\top} \boldsymbol{w}-\boldsymbol{D}\right)-F_{\ell} \leq 0, \quad \forall \ell \in \mathcal{L}$,

$-F_{\ell}-\boldsymbol{M}_{(\ell, \cdot)}\left(\boldsymbol{H}_{\mathrm{G}}^{\top} \boldsymbol{p}+\boldsymbol{H}_{\mathrm{J}}^{\top} \boldsymbol{w}-\boldsymbol{D}\right) \leq 0, \quad \forall \ell \in \mathcal{L}$,

2) KKT conditions:

$C_{g}+\gamma^{(36 \mathrm{~b})}-\gamma_{g}^{(36 \mathrm{c})}+\gamma_{g}^{(36 \mathrm{~d})} \ldots$

$+\sum_{\ell \in \mathcal{L}}\left(\gamma_{\ell}^{(36 \mathrm{~g})}-\gamma_{\ell}^{(36 \mathrm{~h})}\right) \boldsymbol{M}_{(\ell, \cdot)} \boldsymbol{H}_{\mathrm{G}}^{\top} \mathbb{1}_{g}=0, \quad \forall g \in \mathcal{G}$,

$\gamma^{(36 \mathrm{~b})}-\gamma^{(36 \mathrm{e})}+\gamma^{(36 \mathrm{f})} \ldots$

$+\sum_{\ell \in \mathcal{L}}\left(\gamma_{\ell}^{(36 \mathrm{~g})}-\gamma_{\ell}^{(36 \mathrm{~h})}\right) \boldsymbol{M}_{(\ell, \cdot)} \boldsymbol{H}_{\mathrm{J}}^{\top} \mathbb{1}_{j}=0, \quad \forall j \in \mathcal{J}$

$\sum_{g \in \mathcal{G}} p_{g}+\sum_{j \in \mathcal{J}} w_{j}-\sum_{n \in \mathcal{N}} D_{n}=0$

$0 \geq \underline{P}_{g}+r_{g}^{-, *}-p_{g} \quad \perp \quad \gamma_{g}^{(36 \mathrm{c})} \geq 0, \quad \forall g \in \mathcal{G}$,

$0 \geq p_{g}-\bar{P}_{g}+r_{g}^{+, *} \quad \perp \quad \gamma_{g}^{(36 \mathrm{~d})} \geq 0$,

$\forall g \in \mathcal{G}$,

$0 \geq-w_{j} \quad \perp \quad \gamma_{j}^{(36 \mathrm{e})} \geq 0$,

$0 \geq w_{j}-\widehat{W}_{j} \quad \perp \quad \gamma_{j}^{(36 \mathrm{f})} \geq 0$,

$\forall j \in \mathcal{J}$ 


$$
\begin{array}{cc}
0 \geq \boldsymbol{M}_{(\ell, \cdot)}\left(\boldsymbol{H}_{\mathrm{G}}^{\top} \boldsymbol{p}+\boldsymbol{H}_{\mathrm{J}}^{\top} \boldsymbol{w}-\boldsymbol{D}\right)-F_{\ell} & \forall \ell \in \mathcal{L}, \\
\perp \gamma_{\ell}^{(36 \mathrm{~g})} \geq 0, & \\
0 \geq-F_{\ell}-\boldsymbol{M}_{(\ell, \cdot)}\left(\boldsymbol{H}_{\mathrm{G}}^{\top} \boldsymbol{p}+\boldsymbol{H}_{\mathrm{J}}^{\top} \boldsymbol{w}-\boldsymbol{D}\right) & \forall \ell \in \mathcal{L} . \\
\perp \gamma_{\ell}^{(36 \mathrm{~h})} \geq 0, &
\end{array}
$$

\section{APPENDIX B}

The master problem of the multi-cut Bender's decomposition scheme at iteration $\eta$ is formulated as

$$
\begin{array}{lll}
\min _{\Xi_{\mathrm{MP}}} & \mathcal{C}_{\mathrm{R}}+\mathcal{C}_{\mathrm{D}}+\sum_{s \in \mathcal{S}} \pi_{s} \vartheta_{s} & \\
\text { s.t. } & (26)-(35), & \text { KKT of zonal reserve market } \\
& (37)-(45), & \text { KKT of day-ahead market } \\
& (20 \mathrm{~d}), & \text { Zonal reserve requirements, } \\
& (5)-(9), & \text { Grid partitioning, } \\
& (15)-(19), & \text { Capacity allocation, } \\
\vartheta_{s} \geq \vartheta_{0} & \forall s \in \mathcal{S} \\
\vartheta_{s} \geq \mathcal{C}_{\mathrm{B}, s}^{(k)}+\sum_{g} \gamma_{g, s}^{(47 \mathrm{~b})^{(k)}} & \left(r_{g}^{+}-r_{g}^{+;(k)}\right) \ldots \\
+\sum_{g} \gamma_{g, s}^{(47 \mathrm{c})^{(k)}}\left(r_{g}^{-}-r_{g}^{-;(k)}\right)+\sum_{g} \gamma_{g, s}^{(47 \mathrm{~d})^{(k)}}\left(p_{g}-p_{g}^{(k)}\right) \ldots \\
+\sum_{j} \gamma_{j, s}^{(47 \mathrm{e})^{(k)}}\left(w_{j}-w_{j}^{(k)}\right), \forall s \in \mathcal{S}, \forall k=1, \ldots, \eta-1
\end{array}
$$$$
\text { where } \gamma^{(*)^{(k)}} \text { are the dual variables of constraints }(*) \text { in the }
$$
sub-problem, whose formulation for scenario $s=s^{\prime}$ and iteration $\eta$ is the following

$$
\begin{aligned}
& \min _{\Xi_{\mathrm{SP}}} \mathcal{C}_{\mathrm{B}, s^{\prime}}=\sum_{g \in \mathcal{G}} C_{g}\left(p_{g, s^{\prime}}^{+}-p_{g, s^{\prime}}^{-}\right) \\
& +\sum_{j \in \mathcal{J}} C^{\mathrm{ct}} w_{j, s^{\prime}}^{\mathrm{ct}}+\sum_{n \in \mathcal{N}} C^{\mathrm{sh}} d_{n, s^{\prime}}^{\mathrm{sh}} \\
& \text { s.t. } r_{g}^{+}=r_{g}^{+;(\eta)} \\
& r_{g}^{-}=r_{g}^{-;(\eta)} \\
& p_{g}=p_{g}^{(\eta)} \\
& w_{j}^{+}=w_{j}^{(\eta)} \\
& \text { (3b) - (3h), } \\
& : \gamma_{g, s^{\prime}}^{(47 b)} \\
& : \gamma_{g, s^{\prime}}^{(47 \mathrm{c})^{(\eta)}} \\
& : \gamma_{g, s^{\prime}}^{(47 \mathrm{~d})^{(\eta)}} \\
& : \gamma_{j, s^{\prime}}^{(47 \mathrm{e})^{(\eta)}}
\end{aligned}
$$

where first-stage decision variables $r_{g}^{+}, r_{g}^{-}, p_{g}$ and $w_{j}$ are fixed to the solution of the master problem at the current iteration. As the problem has complete recourse, no need for feasibility cuts is required and $\mathcal{S}$ new optimality cuts are added to the master problem at each iteration. The algorithm converges to a solution when the condition $\left|\sum_{s} \pi_{s} \vartheta_{s}-\sum_{s} \pi_{s} \mathcal{C}_{\mathrm{B}, s}^{(\eta)}\right| \leq \varepsilon$ is satisfied for a predefined tolerance $\varepsilon$.

\section{ACKNOWLEDGMENT}

The authors wish to acknowledge the financial support received from the Danish transmission system operator, Energinet, and Ørsted Offshore Wind A/S in the final stages of the project.

\section{REFERENCES}

[1] J.M. Morales, A.J. Conejo and J. Pérez-Ruiz, "Economic Valuation of Reserves in Power Systems With High Penetration of Wind Power," IEEE Trans. on Power Syst., vol. 24, no. 2, pp. 900-910, 2009.

[2] K. Van den Bergh, R. B. Hytowitz, K. Bruninx, E. Delarue, W. Dhaeseleer, and B. F. Hobbs, "Benefits of coordinating sizing, allocation and activation of reserves among market zones," Electr. Pow. Syst. Res., vol. 143, pp. 140-148, 2017.

[3] K. Van den Bergh, K. Bruninx, and E. Delarue, "Cross-border reserve markets: network constraints in cross-border reserve procurement," Energy Policy, vol. 113, pp. 193-205, 2018.

[4] K. Van Den Bergh and E. Delarue, "An improved method to calculate injection shift keys," Electr. Pow. Syst. Res., vol. 134, no. July, pp. 197204, 2016.

[5] B. Park, Z. Zhou, A. Botterud, and P. Thimmapuram, "Probabilistic zonal reserve requirements for improved energy deliverability with wind power," IEEE Trans. on Power Syst., pp. 1-1, 2020.

[6] F. D. Galiana, F. Bouffard, J. M. Arroyo, and J. F. Restrepo, "Scheduling and pricing of coupled energy and primary, secondary, and tertiary reserves," Proc. of the IEEE, vol. 93, no. 11, pp. 1970-1983, Nov 2005.

[7] A. Papavasiliou, S. S. Oren, and R. P. O'Neill, "Reserve Requirements for Wind Power Integration : A Scenario-Based Stochastic Programming Framework," IEEE Trans. on Power Syst., vol. 26, no. 4, pp. 2197-2206, 2011.

[8] A. Papavasiliou and S. S. Oren, "Multiarea stochastic unit commitment for high wind penetration in a transmission constrained network." Oper. Res., vol. 61, no. 3, pp. 578 - 592, 2013.

[9] J. M. Morales, M. Zugno, S. Pineda, and P. Pinson, "Electricity market clearing with improved scheduling of stochastic production," Eur. J. Oper. Res, vol. 235, no. 3, pp. 765-774, 2014.

[10] V. Dvorkin, S. Delikaraoglou, J.M. Morales, "Setting reserve requirements to approximate the efficiency of the stochastic dispatch," IEEE Trans. on Power Syst., vol. 34, no. 2, pp. 1524-1536, 2019.

[11] S. Delikaraoglou, P. Pinson, "Optimal allocation of HVDC interconnections for exchange of energy and reserve capacity services," Energy Syst., vol. 10, no. 3, pp. 635-675, 2019.

[12] N. Fan, D. Izraelevitz, F. Pan, P. M. Pardalos, and J. Wang, "A mixed integer programming approach for optimal power grid intentional islanding," Energy Syst., vol. 3, no. 1, pp. 77-93, 2012.

[13] M. Golari, N. Fan, and J. Wang, "Two-stage stochastic optimal islanding operations under severe multiple contingencies in power grids," Electr. Pow. Syst. Res., vol. 114, pp. 68-77, 2014.

[14] A. Kumar, S. C. Srivastava, and S. N. Singh, "A zonal congestion management approach using real and reactive power rescheduling," IEEE Trans. on Power Syst., vol. 19, no. 1, pp. 554-562, Feb 2004.

[15] F. Wang and K. W. Hedman, "Reserve zone determination based on statistical clustering methods," in Proc. NAPS, Sep. 2012, pp. 1-6.

[16] F. Wang and K. W. Hedman, "Dynamic reserve zones for day-ahead unit commitment with renewable resources," IEEE Trans. on Power Syst., vol. 30, no. 2, pp. 612-620, 2015.

[17] Y. Chen, P. Gribik, and J. Gardner, "Incorporating Post Zonal Reserve Deployment Transmission Constraints Into Energy and Ancillary Service Co-Optimization," IEEE Trans. on Power Syst., vol. 29, no. 2, pp. 537549, 2014.

[18] A. Ahmadi-Khatir, M. Bozorg, and R. Cherkaoui, "Probabilistic spinning reserve provision model in multi-control zone power system," IEEE Trans. on Power Syst., vol. 28, no. 3, pp. 2819-2829, Aug 2013.

[19] J. D. Lyon, S. Member, F. Wang, S. Member, and K. W. Hedman, "Market Implications and Pricing of Dynamic Reserve Policies for Systems With Renewables," IEEE Trans. on Power Syst., vol. 30, no. 3, pp. 1593-1602, 2015.

[20] J. D. Lyon, M. Zhang, and K. W. Hedman, "Locational reserve disqualification for distinct scenarios," IEEE Trans. on Power Syst., vol. 30, no. 1, pp. 357-364, Jan 2015.

[21] Y. T. Gebrekiros, G. L. Doorman, H. Farahmand, and S. Jaehnert, "Benefits of cross-border reserve procurement based on pre-allocation of transmission capacity," Proc. IEEE Grenoble PowerTech, pp. 1-6, 2013.

[22] ENTSO-E, "Principles for determining the transfer capacities in the nordic power market," December 2018. [Online]. Available: https://www.nordpoolspot.com/globalassets/downloadcenter/tso/principles-for-determining-the-transfer-capacities.pdf

[23] L. Fournié, C. Andrey, J. Hentschel, G. Wilkinson, "Integration of electricity balancing markets and regional procurement of balancing reserves," 2016. [Online]. Available: https://ec.europa.eu/energy/studies/integration-electricitybalancing-markets-and-regional-procurement-balancing-reserves_en 
[24] R. Domínguez, G. Oggioni, and Y. Smeers, "Reserve procurement and flexibility services in power systems with high renewable capacity: Effects of integration on different market designs," Int. J. Elec Power, vol. 113, no. June, pp. 1014-1034, 2019.

[25] K. Poplavskaya, G. Totschnig, F. Leimgruber, G. Doorman, G. Etienne, and L. de Vries, "Integration of day-ahead market and redispatch to increase cross-border exchanges in the european electricity market," Appl. Energy, vol. 278, p. 115669, 2020.

[26] B. Dilkina and C. P. Gomes, "Solving connected subgraph problems in wildlife conservation," in Proc. CPAIOR, pp. 102-116, 2010.

[27] F. Trespalacios and I. Grossmann, "Review of mixed-integer nonlinear and generalized disjunctive programming methods," Chemie-IngenieurTechnik, vol. 86, no. 7, pp. 991-1012, 2014, cited By 72.

[28] S. Pineda and J. M. Morales, "Solving linear bilevel problems using bigms: Not all that glitters is gold," IEEE Trans. on Power Syst., vol. 34, no. 3, pp. 2469-2471, 2019.

[29] A. J. Conejo, E. Castillo, R. Mínguez, and R. García-Bertrand, Decomposition techniques in mathematical programming: Engineering and science applications. Springer, 2006.

[30] H. Bludszuweit, J. A. Domínguez-Navarro, and A. Llombart, "Statistical analysis of wind power forecast error," IEEE Trans. on Power Syst., vol. 23, no. 3, pp. 983-991, 2008.

[31] G. Papaefthymiou and D. Kurowicka, "Using copulas for modeling stochastic dependence in power system uncertainty analysis," IEEE Trans. on Power Syst., vol. 24, no. 1, pp. 40-49, 2009.

[32] J. Dupačová, G. Consigli, and S. W. Wallace, "Scenarios for Multistage Stochastic Programs," Ann. Oper. Res, vol. 100, no. 1-4, pp. 25-53, 2000.

[33] C. Ordoudis, P. Pinson, and M. Zugno, "An updated version of the IEEE rts 24-bus system for electricity market and power system operation studies," Technical University of Denmark (DTU), pp. pp. 1-5, 2016.

[34] H. Pandzic, Y. Dvorkin, T. Qiu, Y. Wang, and D. Kirschen, "Unit Commitment under Uncertainty - GAMS Models," Library of the Renewable Energy Analysis Lab (REAL). [Online]. Available: http://www.ee.washington.edu/research/real/gams_code.html

[35] "Regulation (eu) 2019/943 of the european parliament and of the council of 5 june 2019 on the internal market for electricity," 2019. [Online]. Available: http://data.europa.eu/eli/reg/2019/943/oj

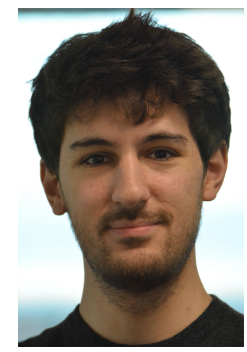

Nicola Viafora (S'18) received the B.Sc in Energy Engineering and the M.Sc in Electrical Energy Engineering from the Università degli Studi di Padova, Padova, Italy, in 2013 and 2016, respectively. He was awarded the Ph.D. degree by the Department of Electrical Engineering at the Technical University of Denmark, Lyngby, Denmark, in 2020. His research interests include optimization and decisionmaking in power systems, uncertainty modelling and dynamic line ratings. He recently joined the Nordic Regional Security Coordinator in Copenhagen, Denmark, focusing on coordinated security analysis.

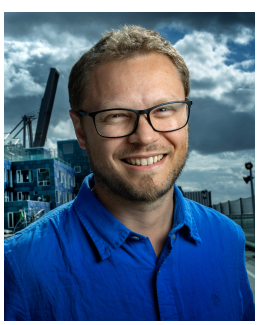

Pierre Pinson (SM'13, F'20) received the M.Sc. degree in applied mathematics from the National Institute for Applied Sciences, Toulouse, France, and the Ph.D. degree in energetics from Ecole des Mines de Paris, Paris, France. He is a Professor with the Centre for Electric Power and Energy, Department of Electrical Engineering, Technical University of Denmark, Lyngby, Denmark, also heading a group focusing on energy analytics and markets. His research interests include forecasting, uncertainty estimation, optimization under uncertainty, decision sciences, and renewable energies. He is the Editor-in-Chief for the International Journal of Forecasting.

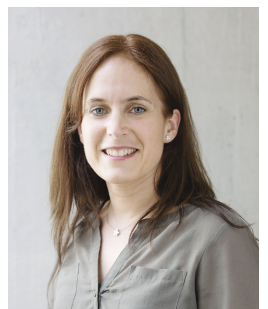

Gabriela Hug (S'05-M'08-SM'14) was born in Baden, Switzerland. She received the M.Sc. degree in electrical engineering in 2004 and the Ph.D. degree in 2008, both from the Swiss Federal Institute of Technology, Zurich, Switzerland. After the Ph.D. degree, she worked with the Special Studies Group of Hydro One, Toronto, ON, Canada, and from 2009 to 2015, she was an Assistant Professor with Carnegie Mellon University, Pittsburgh, PA, USA. She is currently a Professor with the Power Systems Laboratory, ETH Zurich, Zurich, Switzerland. Her research is dedicated to control and optimization of electric power systems.

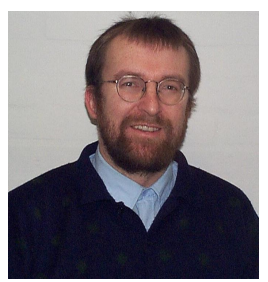

Joachim Holbøll is professor of High Voltage Engineering at Technical University of Denmark (DTU), Department of Electrical Engineering. He received his Ph.D.-degree from DTU in 1992. After some early years with studies within high voltage insulation and electrical phenomena, his main field of research is now high voltage components, their properties and condition in close relation to advanced modelling. Past years focus has been on high voltage transformers, cables and, in general, components' performance under AC, DC and transients. The work is now concentrated on wind energy components and high voltage research with respect to the future power grid. He has been visiting researcher at Ontario Hydro Research, Canada, Royal Institute of Technology in Sweden and Monash University, Australia. He is a Senior Member of IEEE and member of Cigré National Committee of Denmark.

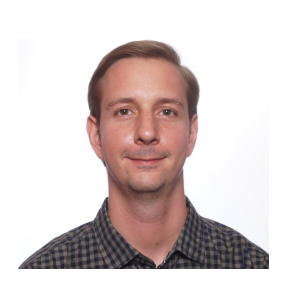

Stefanos Delikaraoglou (S'14-M'18) received the Dipl.-Eng. degree from the School of Mechanical Engineering, National Technical University of Athens, Greece, in 2010 and the M.Sc. degree in Sustainable Energy from the Technical University of Denmark in 2012. He holds a Ph.D. degree awarded in 2016 by the Department of Electrical Engineering at the Technical University of Denmark. From 2017 to 2019 , he was a Postdoctoral researcher with the Power Systems Laboratory at the Swiss Federal Institute of Technology (ETH), Zurich, Switzerland, and he is currently a Postdoctoral Associate with the Laboratory for Information and Decision Systems (LIDS), Massachusetts Institute of Technology (MIT), MA, USA.

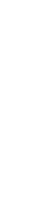

